\title{
Article
}

\section{Effect of a congested match schedule on immune-endocrine responses, technical performance, and session-RPE in elite youth soccer players}

Moreira, A., Bradley, P.S., Carling, C., Arruda, A.F.S., Spigolon, L.M.P., Franciscon, C., and Aoki, M.S.

Available at http://clok.uclan.ac.uk/14720/

Moreira, A., Bradley, P.S., Carling, C. ORCID: 0000-0002-7456-3493, Arruda, A.F.S., Spigolon, L.M.P., Franciscon, C., and Aoki, M.S. (2016) Effect of a congested match schedule on immune-endocrine responses, technical performance, and session-RPE in elite youth soccer players. Journal of Sports Sciences: Science and Medicine in Football, 34 (24). pp. 2255-2261. ISSN 0264-0414

It is advisable to refer to the publisher's version if you intend to cite from the work. http://dx.doi.org/10.1080/02640414.2016.1205753

For more information about UCLan's research in this area go to http://www.uclan.ac.uk/researchgroups/ and search for <name of research Group>.

For information about Research generally at UCLan please go to http://www.uclan.ac.uk/research/

All outputs in CLoK are protected by Intellectual Property Rights law, including Copyright law. Copyright, IPR and Moral Rights for the works on this site are retained by the individual authors and/or other copyright owners. Terms and conditions for use of this material are defined in the policies page. 
This is a pre-proof corrected manuscript, as accepted for publication, of an article published by Taylor \& Francis in Journal of Sports Sciences: Science and Medicine in Football on $8^{\text {th }}$ July, avaliable online: http://www.tandfonline.com/doi/full/10.1080/02640414.2016.1205753

Effect of a Congested Match Schedule on Immune-Endocrine responses, Technical Performance, and Session-RPE in Elite Youth Soccer Players

Running head: Effect of Match Congestion in Youth Soccer

Alexandre Moreira ${ }^{1}$, Paul S. Bradley ${ }^{2}$, Chris Carling ${ }^{3}$, Ademir F.S. Arruda ${ }^{1}$, Leandro M.P. Spigolon ${ }^{4}$, Clovis Franciscon ${ }^{4}$, Marcelo S. Aoki ${ }^{5}$,

${ }^{1}$ Department of Sport, School of Physical Education and Sport, University of Sao Paulo, Sao Paulo, Brazil.

${ }^{2}$ School of Sport, Leeds Beckett University, UK.

${ }^{3}$ Institute of Coaching and Performance, University of Central Lancashire, Preston, UK.

${ }^{4}$ Desportivo Brazil, Porto Feliz, Brazil.

${ }^{5}$ School of Arts, Sciences and Humanities, University of Sao Paulo, Sao Paulo, Brazil

Corresponding author

Dr Alexandre Moreira

School of Physical Education and Sport, University of Sao Paulo, Sao Paulo, Brazil.

Av. Prof. Mello Moraes, 65, Cidade Universitária, São Paulo, Brazil. Zip Code 05508-030. E-mail: alemoreira@usp.br

Telephone: +55-11-30918789

Fax: +55-11-38135091

Key-words: football; testosterone; cortisol; saliva; Immunoglobulin A. 


\begin{abstract}
This study investigated the effects of a congested match schedule ( 7 matches played in 7 days) on steroid hormone concentrations, mucosal immunity, session rating of perceived exertion (S-RPE) and technical performance in 16 elite youth soccer players $(14.8 \pm 0.4 \mathrm{yr} ; 170.6 \pm 9.4 \mathrm{~cm} ; 64.9 \pm 7 \mathrm{~kg})$. No change was observed for salivary cortisol concentration across multiple matches $(p=0.33$; Effect size $[E S]=0.13-0.48)$. In contrast, there was a decrease in salivary testosterone and $\operatorname{Ig} \mathrm{A}$ concentrations $(\mathrm{SIgA})$ from the $1^{\text {st }}$ compared to the last timepoint ( $\mathrm{p}=0.01$ and $0.001, \mathrm{ES}=0.42$ and 0.67 , respectively). The SIgA concentration varied across time points $(\mathrm{p}<0.001)$ with the highest value observed at the $3^{\text {rd }}$ time-point (rest-day) $\left(3^{\text {rd }}\right.$ versus all time-point; ES=0.47-0.73). No changes were observed for S-RPE across matches ( $p>0.05)$. A higher number of tackles and interceptions were observed during the $4^{\text {th }}$ match vs. $1^{\text {st }}$ and $7^{\text {th }}$ matches $(\mathrm{p}<0.001 ; \mathrm{ES}=2.25$ and 1.90, respectively). The present data demonstrate that accumulated fatigue related to participation in a congested match schedule might induce a decrease in testosterone concentration in youth players' and negatively affect their mucosal immunity and capacity to perform certain technical actions.
\end{abstract}

Key-words: football; competition; testosterone; cortisol; saliva; Immunoglobulin A. 


\section{INTRODUCTION}

There is a growing body of literature on the effect of congested match schedules on physical and physiological performance in elite senior soccer players (Dellal et al., 2013; Dupont et al., 2010; Odetoyinbo, Wooster, \& Lane, 2009). However, official youth tournaments in which elite young soccer players participate can also involve congested schedules (Arruda et al., 2015; Buchheit, Horobeanu, MendezVillanueva, Simpson, \& Bourdon, 2011; Rowsell, Coutts, Reaburn, \& Hill-Haas, 2011). While these studies have shown that running activity can be affected due to match congestion, relatively little is known about the potential effect of playing successive matches in a short period on technical performance metrics in youth populations.

This knowledge is important as it have been reported that physical fatigue represented by a reduction in running activity can affect skill-related performance in soccer match-play (Rampinini, Impellizzeri, Castagna, Coutts, \& Wisløff, 2009). Indeed, Stone and Oliver (2009) suggested that resistance to fatigue plays a role in the effectiveness of players' ability to maintain optimal performance and perform precise movements during match play. Rampinini et al. (2009) reported a decline in technical and physical performance when comparing first and second halves of play in elite senior players. In contrast, Carling and Dupont (2011) demonstrated that neither physical nor skill-related performance was affected across three consecutive games within a period of $\leq 7$ days in an elite senior soccer team. Research is warranted to examine physical and technical performance metrics in elite youth soccer populations.

Similarly, the effects of a very congested match schedule on neuroimmune-endocrine responses of youth players are still relatively unclear. Findings from previous studies suggest that successive matches and periods of increased 
training load may affect psycho-physiological responses in youth players. Rowsell et al. (2009) demonstrated increased concentrations of muscle damage markers (creatine kinase and lactate dehydrogenase) in male junior players completing four competitive matches in 4 days with $\sim 24 \mathrm{~h}$ between matches. Freitas et al. (2014) provided evidence that intensive periods of training may lead to an increase in salivary cortisol concentration but not stress tolerance in elite youth soccer players. In addition, Mortatti et al. (2012) reported that a decrement in mucosal immunity, as measured by salivary $\operatorname{IgA}$ concentration, can lead to a greater incidence of upper respiratory tract illness (URTI) in elite Under 19 soccer players monitored in a series of 7 matches in 20 days.

Given the importance of extending current knowledge in elite soccer regarding the impact of successive matches played in a short time interval using a more holistic and integrated approach (Carling et al., 2015), the aim of this study was to analyse the effects of a congested match schedule on steroid hormones concentrations (salivary testosterone and cortisol), mucosal immunity, technical performance parameters, and session rating of perceived exertion (S-RPE) in elite Under-15 players during a major national tournament. It was hypothesized that successive matches played in a short time interval would negatively affect technical performance, and simultaneously lead to negative changes in neuroimmune-endocrine parameters.

\section{METHODS}

Participants

Eighteen elite Under-15 Brazilian soccer players initially volunteered to participate in this study. Of these, 16 outfield players participated in matches and 
were thus considered for further analysis (age $14.8 \pm 0.4 \mathrm{yr} ; 1.5 \pm 0.7 \mathrm{yr}$ from peak height velocity; height $170.6 \pm 9.4 \mathrm{~cm}$ and body mass $64.9 \pm 7 \mathrm{~kg}$ ). Players typically participated in $\sim 15 \mathrm{~h}$ of training per week (5-8 soccer training sessions, 3-4 strength training sessions, 1-2 conditioning sessions) and a single match. Informed consent was obtained from each player and their parents or guardians and the study was approved by the appropriate institutional ethics committee. All players underwent a thorough medical assessment to verify their health status prior to participation and were free from illness or injury at the time of this study.

\section{Procedures}

Player performances in a reference team were monitored during an annual national tournament in which seven matches were played in seven days during the second week of May. The first two matches were played on the first day of the competition (two halves of $20 \mathrm{~min}$ interspersed by $10 \mathrm{~min}$ rest). The $1^{\text {st }}$ match of the day was played at 9:00 am and the $2^{\text {nd }}$ at 3:00 $\mathrm{pm}$. The $3^{\text {rd }}$ and $4^{\text {th }}$ matches were played on the second day and the $5^{\text {th }}$ and $6^{\text {th }}$ matches of the tournament were played on the sixth day of the week, after a 3 day lay-off. On the final day of the tournament, there was only one match, played at 9:00 am. The mean $( \pm \mathrm{SD})$ temperature and humidity during the tournament were, $20^{\circ} \mathrm{C}( \pm 4)$ and $77 \%( \pm 5)$, respectively. The reference team won matches 1-4, drew matches 5 and 6 and lost match 7 (Table 1). No post-match recovery strategies were implemented during this study period. Saliva samples were collected on mornings in a fasted state (no food 6-7 $\mathrm{hr}$ before sampling) on each match day. Players were woken up approximately 15 min prior to sampling. Saliva sampling was also undertaken on day 3 (without match) at the same time of day (fasted state). Session rating of 
perceived exertion (S-RPE) was used to quantify the perceived intensity of each match (Foster, 1998). All players were familiar with these procedures.

***INSERT TABLE 1 NEAR HERE***

\section{Video Analysis and Technical Performance}

Video recordings were collected using two cameras (Panasonic, $60 \mathrm{~Hz}$ frequency acquisition). One was located $15 \mathrm{~m}$ above and to one side of the long axis of the pitch, and the other was placed $5 \mathrm{~m}$ to one side of the pitch to facilitate the player's identification. SportsCode GameBreaker Plus 10 software (Sportstec, Sydney, Australia) was used to code match performance. The inter- and intrareliability was found to be excellent when analyzing two trials for each match using two experienced analysts. The Kappa values for the analysed variables ranged between $0.90-0.98$ (intra-observer) to $0.82-0.91$ (inter-observers). The technical events were chosen to fall in line with those used in previous research literature (Rampinini et al., 2009; Waldron \& Worsfold, 2010) and included attacking and defending variables. The frequencies of the following technical actions were recorded for the reference team as a whole and each respective opponent:

- Involvements with the ball: all situations where the player was in contact with the ball.

- Goal attempts: number of attempts to score a goal.

- Total passes: number of short and long foot passes performed by a player. 
- Successful (completed) passes: number of short and/or long passes performed by a player and successfully received by another player of his own team.

- Total headers: number of times where a player touched the ball with his head.

- Tackles and interceptions: number of situations where a player contested the ball with an opponent player irrespective of whether these situations involved or not clear physical contact between players.

- Total involvements: sum of all assessed technical actions.

Attacking variables included: involvements with the ball, goal attempts, passes (total passes and successful [completed] passes); defending variables included: tackles and interceptions made. Headers were included but not classified according to whether these were attacking or defending actions. Descriptive data for the assessed team as a whole (total number of team technical actions) and its opponents are presented.

\section{Saliva Collection and Analysis}

All players remained in a seated position, with eyes open, head tilted slightly forward, and making minimal orofacial movement during saliva collection. Unstimulated saliva was collected in a preweighed sterile $15 \mathrm{~mL}$ centrifuge tube over $5 \mathrm{~min}$ and stored at $-80^{\circ} \mathrm{C}$ until assayed. The tubes were re-weighed before analysis, so that saliva volume could be estimated with saliva density assumed to be $1.00 \mathrm{~g} \cdot \mathrm{mL}^{-1}$. Salivary flow rate was determined by dividing the volume of saliva collected by the duration of the sampling period. After thawing and centrifugation $\left(10,000 \mathrm{~g}\right.$ for $10 \mathrm{~min}$ at $\left.4^{\circ} \mathrm{C}\right)$, the samples were tested for cortisol, 
testosterone and salivary $\operatorname{Ig} \mathrm{A}(\mathrm{SIgA})$ concentrations in duplicate using enzymelinked immunosorbent assays (ELISA, Salimetrics ${ }^{\mathrm{TM}}$ ) in accordance with standard procedures (Freitas et al., 2014; Moreira et al., 2013; Mortatti et al., 2012). The SIgA secretion rate $\left(\mathrm{SIgA}_{\text {rate }}\right.$ - micrograms per minute) was calculated by multiplying the absolute $\mathrm{SIgA}_{\mathrm{abs}}$ by salivary flow rate (milliliters per minute), in accordance with the procedure adopted by Mortatti et al. (2012). The mean intraassay coefficient of variation for cortisol, testosterone, and SIgA concentrations was $3.9,4.5$, and $5.6 \%$, respectively.

\section{Match Intensity}

In order to quantify match intensity, the S-RPE was assessed after each match (Foster, 1998). Each player rated the match intensity using the CR-10 sliding scale 30 min post-match. This method has been shown to be a valid method for monitoring load in young players (Freitas et al., 2014; Mortatti et al., 2012).

\section{Statistical analyses}

Data were analyzed using statistical software (version 12.0, StatSoft). Data normality was assessed by the Shapiro-Wilk's test and homoscedasticity by Levene's test. The technical performance data were not normally distributed and therefore the Friedman test was used to detect differences between matches. A Wilcoxon test with a Bonferroni adjustment was used to verify differences. The Friedman test was also used to compare S-RPE across the 7 matches. ANOVAs with repeated measures were used to compare cortisol, testosterone, and SIgA concentrations (absolute concentration and SIgA rate) in a fasted state across matches and the day without match ( $3^{\text {rd }}$ day of the study). Fisher's LDS post hoc test was used when a significance was observed. Statistical significance was set at 
$\mathrm{p} \leq 0.05$. Effect size $(\mathrm{ES})$ values of $0.20-0.49,0.50-0.79$, and $\geq 0.8$ were considered to represent small, medium, and large differences between values respectively (Cohen, 1988).

\section{RESULTS}

Figure 1 illustrates resting and fasted salivary measures across the investigation period. No change was observed for salivary cortisol concentration across multiple matches $(\mathrm{F}=1.17 ; \mathrm{p}=0.33 ; \mathrm{ES}=0.13-0.48$ [trivial to small]; Figure $1 \mathrm{~A})$. However, there was a significant change in testosterone concentration across the investigated period $(F=2.97 ; p=0.02)$. Post hoc tests showed a decrease on the $3^{\text {rd }}$ compared to the $1^{\text {st }}$ collection time-point $(\mathrm{p}=0.007 ; \mathrm{ES}=0.47$ [small]); an increase in testosterone concentration from the $3^{\text {rd }}$ to the $4^{\text {th }}$ collection time-point $(\mathrm{p}=$ 0.003; ES=0.36 [small]; Figure 1B); and a decrease in concentration when comparing the first with the last time-point $(\mathrm{p}=0.01$; $\mathrm{ES}=0.42$ [small]; Figure 1B). A significant change in SIgA concentration was also observed across timepoints $(\mathrm{F}=4.21 ; \mathrm{p}=0.004)$. A decrease in $\mathrm{S} I g \mathrm{~A}$ concentration was reported between the first and last time-point ( $\mathrm{p}<0.001$; $\mathrm{ES}=0.67$ [medium]; Figure 1C). The SIgA concentration at the $3^{\text {rd }}$ time-point (without matches) was different (higher value) compared to all other time-points except the first time-point. In addition, SIgA concentration was higher on the day without matches in comparison to all other time-points $(\mathrm{p}<0.001$ for all time-point comparisons; ES $=0.47$ [small], 0.48[small], 0.73 [medium], for $3^{\text {rd }}$ vs $2^{\text {nd }}, 3^{\text {rd }}$ vs. $4^{\text {th }}$ and $3^{\text {rd }}$ vs $5^{\text {th }}$, respectively). No difference was evident for $\operatorname{SIgA}$ rate $(\mathrm{F}=0.64 ; \mathrm{p}=0.63$; $\mathrm{ES}=0.03-0.37$ [trivial-small]; Figure 1D) or saliva flow rate in a fasted state $(\mathrm{F}=$ $0.85 ; \mathrm{p}=0.50 ; \mathrm{ES}=0.03-0.10$ [trivial] $)$. No change was observed for S-RPE across the experimental period $\left(\chi^{2}=10.83 ; \mathrm{p}=0.09\right)$. The mean and SD for S-RPE values 
across the matches were: $7.7 \pm 0.4,7.7 \pm 0.6,7.1 \pm 1.2,8.2 \pm 0.7,7.2 \pm 1.3,8.0 \pm$ $0.8,7.7 \pm 0.8$, for matches $1,2,3,4,5,6$, and 7 , respectively.

***INSERT FIGURE 1 NEAR HERE***

Technical performance data are presented in figure 2 and table 2. Figure 2 shows the defending variables which were the only technical parameters that changed over the 7 played matches. A significant change in total tackles and interceptions was observed $\left(\chi^{2}=17.24 ; p=0.008\right)$. A higher value for tackles and interceptions during the $4^{\text {th }}$ match (second match played on the second day of the competition) was observed compared to the $1^{\text {st }}(13.1 \pm 4.8$ vs $4.2 \pm 3.1$; ES $=2.25$ [large] $)$ and $7^{\text {th }}$ matches (vs $5.6 \pm 2.8 ; \mathrm{ES}=1.90$ [large]). Moreover, successful tackles and interceptions also changed during the congested match schedule $\left(\chi^{2}=12.47\right.$; $\mathrm{p}=0.05)$. Successful tackles and interceptions were decreased when comparing values for the $4^{\text {th }}$ vs. the $7^{\text {th }}$ match $(5.0 \pm 2.7$ vs $1.0 \pm 1.5 ; \mathrm{ES}=1.90$ [large] $)$ and between the $5^{\text {th }}(4.0 \pm 2.2)$ and the $7^{\text {th }}$ matches $(E S=0.41$ [trivial] $)$. No change was observed for any other technical performance parameters $(\mathrm{p}>0.05)$. Table 2 shows the total teams occurrence of technical performance parameters over the 7 assessed matches. A higher number of total tackles and interceptions for the $3^{\text {rd }}$ and $4^{\text {th }}$ matches compared to all other matches was observed. The opponent team was the same (same players in the team) for the $3^{\text {rd }}$ and last match (please see Table 1). During the former this team performed 163 tackles and interceptions against the reference team (vs 108), while 75 tackles and interceptions (vs 71) were performed during the final match. 
***INSERT TABLE 2 NEAR HERE***

\section{DISCUSSION}

The aim of this study was to analyze the effects of a congested fixture schedule on steroid hormone concentrations, mucosal immunity, S-RPE and technical match performance parameters in Under-15 players during a major national tournament. No changes in salivary cortisol concentration were observed over the congested match schedule; however, there was a significant decrease in the salivary concentration of testosterone (small ES) and SIgA (moderate ES) from the beginning to the end of the competition. Despite changes in resting testosterone and $\operatorname{SIg} \mathrm{A}$, there was no observed change in the session-RPE score across matches. Regarding technical performance data, defensive performance changed across the competition but attacking performance was unaffected.

The observed resting salivary cortisol response in the present study is in agreement with Mortatti et al. (2012). The authors investigated the subsequent impact of a competition and training period (7 matches over a 20-day period) on resting hormonal and immune parameters in young players from a reference team. The authors reported no alteration in salivary cortisol concentration, despite a progressive increment in internal training load. Given the present results and those reported by Mortatti et al. (2012) it could be suggested that both a short-congested period ( 7 matches in 7 days with short match halves duration) and a longer (7 matches in 20 days with long match halves duration) did not affect significantly the HPA axis responses of youth soccer players, at least, considering their resting cortisol values. The absence of a significant change in resting cortisol during the present match schedule in youth players might suggest that this congested period of matches does not represent a higher physiological stimulus than the habitual 
training schedule of these players. In addition, the absence of changes in resting salivary cortisol maybe, at least in part, attributed to the high-fitness level of the assessed players and to their habitual high daily and weekly-volume training schedule.

Nevertheless, the cortisol response to the congested match schedule should be viewed with caution. In the present study only resting salivary concentration was examined. Some authors have proposed that the examination of exercise induced hormonal responses rather than resting level of hormones may offer a better scenario when regarding hormonal changes, for example during training or even during states of non-functional overreaching and syndrome of overtraining (Hough et al., 2013; Meeusen et al., 2004; 2010). However, it is reasonable to suggest that the practical usefulness of using this approach to assess hormonal changes during a fixture congested match study may be limited.

In contrast to cortisol responses, a significant change in testosterone concentration was observed notably on the $3^{\text {rd }}$ day of collection (morning of the day without match) compared to the $1^{\text {st }}$ and the $4^{\text {th }}$ collection day. A decrease in testosterone concentration has previously been reported after different types of exercise, lasting for hours to days (Peñailillo, Maya, Niño, Torres, \& ZbindenFoncea, 2015; Urhausen and Kindermann, 1987). Peñailillo et al. (2015) for example, compared changes in testosterone concentration from pre- to post-match (5-10 min after match), and demonstrated that salivary testosterone decreased by $\sim 30 \%$ after a soccer match in elite soccer players. The authors also reported that players who showed smaller decreases in testosterone level had covered more distance and illustrated less pronounced alterations to their immune function. While the mechanisms underlying such decrements are beyond the scope of the present investigation, previous observations have nevertheless suggested potential 
mechanisms: 1) a disturbance in gonadotrophin-releasing hormone $(\mathrm{GnRH})$ and testicular luteinising hormone ( $\mathrm{LH})$ pulsatile release; 2) a desensitizing effect on LH receptors; 3) an increased metabolic rate of testosterone and 4) a catecholamine-induced increase in the testicular vascular resistance (Urhausen, Gabriel, \& Kindermann, 1995).

The current results suggest that the successive matches played during the first 2 days of the competition (4 matches in 2 days) potentially affected resting testosterone concentration. Nevertheless, after a recovery period (the time between the $3^{\text {rd }}$ and $4^{\text {th }}$ collection days) the players' testosterone concentration returned to initial levels (higher values at the $4^{\text {th }}$ time-point compared to the $3^{\text {rd }}$ ). The accumulated fatigue potentially generated by the congested competitive schedule seems to lead to a decrement in testosterone as a decrease was also observed on the $5^{\text {th }}$ collection day.

Previous research found that testosterone concentration was affected by tiredness and the magnitude of fatigue (Maso, Lac, Filaire, Michaux, \& Robert, 2004). The authors suggested that a low testosterone level could be used a marker of fatigue, and might reflect the residual effect of a previous accumulated training load and an insufficient recovery period. Thus, the lower salivary testosterone induced by the congested competition may reflect accumulation of fatigue linked to insufficient recovery intervals. In addition, Papadopoulos et al. (2014) observed a decrease in testosterone concentration during a week leading to competition as compared to a control (noncompetition) week, in competitive swimmers (11-15 yr old). Based on previous findings reported by Maso et al. (2004) and Papadopoulos et al. (2014), it seems plausible that the observed testosterone response in the present study is associated to the accumulative fatigue generated during the congested fixture competition. This finding is novel and adds important 
information to the literature regarding the effect of a short-term congested match schedule on salivary testosterone concentration in youth players, and the use of this measure to monitor fatigue in youth players.

Results showed that mucosal immunity was also affected over the congested competitive period. A decreases in SIgA was observed when comparing the first and the final saliva collection time-point (fasted state). It is noteworthy that the mean SIgA value at the $3^{\text {rd }}$ time-point was significantly higher than all other saliva collection time-points. An insufficient recovery period between successive training sessions can cause suppression of the immune system (Bishop \& Gleeson, 2009). Hence it is reasonable to suggest that the increased stress related to the assessed congested fixture schedule on youth players may have compromised players' mucosal immunity. This result suggests that the additional stress of participating in a congested match schedule competition may lead to changes in mucosal immune function in youth players. A significant change in SIgA concentration was reported by Nazem et al. (2011) in young female handball players. The authors reported a decrement in SIgA concentration following matches considered by the players as 'hard' (perceived difficulty of the matches was rated by players). Collectively, the results from the present study and those by Nazem et al. (2011), suggest that additional factors, such as match intensity, competitive anxiety, perceived difficulty of the matches, pressure to perform well, and successive matches, could impose a high level of stress on young team sports players, leading therefore to alterations in their mucosal immunity.

During the present congested match schedule period, S-RPE values were unchanged. However, it is noteworthy that despite the unchanged S-RPE values over the congested match schedule, the matches were played in a high intensity 
zone ( $\geq$ 7; Lovell, Sirotic, Impellizzeri, \& Coutts, 2013; Moreira et al., 2015) demonstrating the high demands placed on the players. This result suggests that despite the likely accumulated fatigue represented by the results from testosterone and SIgA responses, the players might have employed pacing strategies in order to regulate their efforts to avoid fatigue and consequently their perceived effort was unaltered (Bradley \& Noakes, 2013). Whilst, the absence of data on players' running activity is a limitation of the present study for discussing whether the assessed soccer players employed some form of pacing strategy to avoid fatigue, this possibility should be mentioned, although it is evidently speculative. Indeed, it is worth mentioning that the presence of a pacing strategy in young team players has previously been reported (Sampson, Fullagar, \& Gabbett, 2014), and that schoolchildren at a more advanced stage of cognitive development (Under 14) exhibited the well-known U-shaped pacing strategy during the first $15 \%$ and last $20 \%$ of a 4-min run (Micklewright et al., 2012).

Contrary to the hypothesis, a congested match schedule did not affect most of the technical performance parameters assessed in the present study. However, it is worth noting that there was a change in defensive actions, which might be associated to the fixture congestion. This assumption may be reinforced by the fact that these actions were also affected for the opponent teams in the competition. Analysis of the descriptive data of the opponents showed a higher number of tackles and interceptions for the $3^{\text {rd }}$ and $4^{\text {th }}$ matches (163 and 130 tackles and interceptions for the opponent teams, respectively), whilst only 75 tackles and interceptions were observed for the final match. It is worth noting that the opponent team was the same for the $3^{\text {rd }}$ and last match (Table 1). Additionally, it should be highlighted that there was a meaningful decrement ( $3^{\text {rd }}$ vs last match) 
regarding defensive parameters for the assessed team and the opponent team which in turn affected their total involvements.

One potential explanation for the decrement in defensive actions may be the accumulated fatigue associated to the congested competition schedule. Folgado, Duarte, Marques, and Sampaio (2015) speculated that the lower percentage of dyadic synchronization of players observed during their study may be explained by accumulated mental fatigue that impairs a players' capacity to be synchronized with teammates during periods of low-intensity. It is noteworthy that a meaningful reduction in the frequency of defensive actions of the reference players was observed for the last two matches (vs $4^{\text {th }}$ match) of the competition. Notably, the $6^{\text {th }}$ match ended tied and the assessed team lost the final match of the competition. In addition, as a decrease in the salivary testosterone was also observed, it could be inferred that a fatigue state would have actually impaired the capacity of the player to perform, notably in executing defensive actions.

While this is a novel and interesting finding of the effect of a congested match schedule on technical performance in youth players, further empirical studies should be conducted to corroborate the assumption that this reduction in the defensive performance might in fact be attributed to the fatigue state. For example, an investigation into the effect of consecutive running simulations on performance in technical skill tests would be pertinent (Carling et al, 2015). Additionally, including psychometric tools (questionnaires) in order to monitor both the perceived fatigue and perceived effort would add important information regarding the effect of a successive soccer physical demand on players' skills. 


\section{CONCLUSION}

The data demonstrate that the congested period impacted upon salivary immune-endocrine, notably on testosterone and SIgA responses. Moreover, a congested period led to a change in defensive but not in attacking performance metrics. The present results suggest that accumulated fatigue induced by a congested schedule resulted in a decrease in testosterone concentration in youth players and negatively affected their mucosal immunity and capacity to perform certain technical actions. Changes in testosterone have been linked to training motivation, performance, training outcomes, and effort during competition; and declines in SIgA have been associated with an increased risk of upper respiratory tract infections. Hence coaches and support staff should adopt strategies to monitor these parameters during training and competition, in order to evaluate players' ability to cope with the demands of athletic training and competition. However, it is important to highlight that the present findings are representative of a very unique congested match schedule for youth players and thus the results should not be generalized to other situations (i.e. normal training week and other kinds of congested schedules competition or senior players).

\section{REFERENCES}

Arruda, A. F., Carling, C., Zanetti, V., Aoki, M. S., Coutts, A. J., \& Moreira, A. (2015). Effects of a very congested match schedule on body load impacts, accelerations, and running measures in youth soccer players. International Journal of Sports Physiology and Performance, 10, 248-252.

Bishop, N., \& Gleeson, M. (2009). Acute and chronic effects of exercise on markers of mucosal immunity. Frontiers in Bioscience, 14, 4444-4456. 
Bradley, P. S., \& Noakes, T. D. (2013). Match running performance fluctuations in elite soccer: Indicative of fatigue, pacing or situational influences? Journal of Sports Sciences, 31, 1627-1638.

Buchheit, M., Horobeanu, C., Mendez-Villanueva, A., Simpson, B. M., \& Bourdon, P. C. (2011). Effects of age and spa treatment on match running performance over two consecutive games in highly trained young soccer players. Journal of Sports Sciences, 29, 591-598.

Carling, C., \& Dupont, G. (2011). Are declines in physical performance associated with a reduction in skill-related performance during professional soccer match-play? Journal of Sports Sciences, 29, 63-71.

Carling, C., Gregson, W., McCall, A., Moreira, A., Wong, D. P., \& Bradley, P. S. (2015). Match running performance during fixture congestion in elite soccer: research issues and future directions. Sports Medicine, 45, 605-613.

Cohen J. (1988). Statistical power analysis for the behavioral sciences. Hillsdale: Lawrence Erlbaum.

Dellal, A., Lago-Peñas, C., Rey, E., Chamari, K., \& Orhant, E. (2013). The effects of a congested fixture period on physical performance, technical activity and injury rate during matches in a professional soccer team. British Journal of Sports Medicine, 49, 390-394.

Dupont, G., Nedelec, M., McCall, A., McCormack, D., Berthoin, S., \& Wisløff, U. (2010). Effect of 2 soccer matches in a week on physical performance and injury rate. The American Journal of Sports Medicine, 38, 1752-1758.

Folgado, H., Duarte, R., Marques, P., \& Sampaio, J. (2015). The effects of congested fixtures period on tactical and physical performance in elite football. Journal of Sports Sciences, 33, 1238-1247.

Foster, C. (1998). Monitoring training in athletes with reference to overtraining 
syndrome. Medicine and Science in Sports and Exercise, 40, 1164-1168.

Freitas, C. G., Aoki, M. S., Franciscon, C. A., Arruda, A. F. S., Carling, C., \& Moreira, A. (2014). Psychophysiological responses to overloading and tapering phases in elite young soccer players. Pediatric Exercise Science, 26, 195-202.

Hough, J., Corney, R., Kouris, A., \& Gleeson, M. (2013). Salivary cortisol and testosterone responses to high-intensity cycling before and after an 11-day intensified training period. Journal of Sports Sciences, 31, 1614-1623.

Lovell, T. W. J., Sirotic, A. C., Impellizzeri, F. M., \& Coutts, A. J. (2013). Factors affecting perception of effort (session rating of perceived exertion) during rugby league training. International Journal of Sports Physiology and Performance, 8, $62-69$.

Maso, F., Lac, G., Filaire, E., Michaux, O., \& Robert, A. (2004). Salivary testosterone and cortisol in rugby players: correlation with psychological overtraining items. British Journal of Sports Medicine, 38, 260-263.

Meeusen, R., Piacentini, M. F., Busschaert, B., Buyse, L., De Schutter G, \& Stray-Gundersen, J. (2004). Hormonal responses in athletes: The use of a two bout exercise protocol to detect subtle differences in (over)training status. European Journal of Applied Physiology, 91, 140-146.

Meeusen, R., Nederhof, E., Buyse, L., Roelands, B., de Schutter, G., \& Piacentini, M. F. (2010). Diagnosing overtraining in athletes using the two-bout exercise protocol. British Journal of Sports Medicine, 44, 642-648.

Micklewright, D., Angus, C., Suddaby, J., St Clair Gibson, A. S. C., Sandercock, G., \& Chinnasamy, C. (2012). Pacing strategy in schoolchildren differs with age and cognitive development. Medicine \& Science in Sports \& Exercise, 44, 362369.

Moreira, A., Coutts, A. J., Moreira, A., Bilsborough, J. C., Sullivan, C. J., 
Cianciosi, M., ... Coutts, A. J. (2015). Training periodization of professional australian football players during an entire australian football league season. International Journal of Sports Physiology and Performance, 10, 566-571.

Moreira, A., Mortatti, A., Aoki, M., Arruda, A., Freitas, C., \& Carling, C. (2013). Role of free testosterone in interpreting physical performance in elite young brazilian soccer players. Pediatric Exercise Science, 25, 186-197.

Mortatti, A., Moreira, A., Aoki, M. S., Crewther, B., Castagna, C., Arruda, A., \& Filho, J. (2012). Effect of competition on salivary cortisol, immunoglobulin A, and upper respiratory tract infections in elite young soccer players. The Journal of Strength and Conditional Reserach, 26, 1396-1401.

Nazem, G., Sharifi, G. R., Taghian, F., Jourkesh, M., Ostoic, S., Calleja-Gonzalez, J., \& Keikhai, B. (2011). The effects of successive official competitions on salivary cortisol and immunogolobulin responses in women handballers. Serbian Journal of Sports Sciences, 5, 67-73.

Odetoyinbo, K., Wooster, B., \& Lane, A. (2009). The effect of a succession of matches on the activity profiles of professional soccer players. In T. Reilly \& F. Korkusuz (Eds.), Science and Football (pp. 182-185). London, United Kingdom: Routledge.

Papadopoulos, E., Muir, C., Russell, C., Timmons, B.W., Falk, B., \& Klentrou, P. Markers of biological stress and mucosal immunity during a week leading to competition in adolescent swimmers. Journal of Immunology Research. Epub 2014, June 12. doi: 10.1155/2014/234565.

Peñailillo, L., Maya, L., Niño, G., Torres, H., \& Zbinden-Foncea, H. (2015). Salivary hormones and $\operatorname{IgA}$ in relation to physical performance in football. Journal of Sports Sciences, 33, 2080-2087.

Rampinini, E., Impellizzeri, F. M., Castagna, C., Coutts, A. J., \& Wisløff, U. 
(2009). Technical performance during soccer matches of the Italian Serie A league: effect of fatigue and competitive level. Journal of Science and Medicine in Sport, 12, 227-233.

Rowsell, G. J., Coutts, A. J., Reaburn, P., \& Hill-Haas, S. (2009). Effects of coldwater immersion on physical performance between successive matches in highperformance junior male soccer players. Journal of Sports Sciences, 27, 565-573. Rowsell, G. J., Coutts, A. J., Reaburn, P., \& Hill-Haas, S. (2011). Effect of postmatch cold-water immersion on subsequent match running performance in junior soccer players during tournament play. Journal of Sports Sciences, 29, 1-6.

Sampson, J.A., Fullagar, H.H., \& Gabbett, T. (2014). Knowledge of bout duration influences pacing strategies during small-sided games. Journal of Sports Sciences, 33, 85-98.

Stone, K. J., \& Oliver, J. L. (2009). The effect of 45 minutes of soccer- specific exercise on the performance of soccer skills. International Journal of Sports Physiology and Performance, 4, 163-175.

Urhausen, A., Gabriel, H., \& Kindermann, W. (1995). Blood hormones as markers of training stress and overtraining. Sports Medicine, 20, 251-276.

Urhausen, A., Kindermann, W. (1987). Behavior of testosterone, sex hormone binding globulin (SHBG), and cortisol before and after a triathlon competition. International Journal of Sports Medicine, 8, 305-308.

Waldron, M., \& Worsfold, P. (2010). Differences in the Game Specific Skills of Elite and Sub-Elite Youth Football Players: Implications for Talent Identification. International Journal of Performance Analysis in Sport, 9-24. 
Table 1. Competition schedule and results

\begin{tabular}{ccccl}
\hline Match & Opponent & Result & $\begin{array}{c}\text { Assessed } \\
\text { team match } \\
\text { outcome }\end{array}$ & $\begin{array}{c}\text { Day of the } \\
\text { investigation; } \\
\text { Period }\end{array}$ \\
\hline First & SE Palmeiras & $1-0$ & Won & $1^{\text {st }}$ Morning \\
Second & Santos FC & $3-2$ & Won & $1^{\text {st }}$ Afternoon \\
Third & SC Internacional & $1-0$ & Won & $2^{\text {nd. }}$ Morning \\
Fourth & Audax & $1-0$ & Won & $2^{\text {nd. }}$ Afternoon \\
Fifth & Bahia EC & $1-1$ & Draw & $6^{\text {th }}$; Morning \\
Sixth & Botafogo RJ & $0-0$ & Draw & $6^{\text {th }}$ Afternoon \\
Seventh & SC Internacional & $0-3$ & Lost & $7^{\text {th }}$; Morning \\
\hline
\end{tabular}


Table 2. Total teams (assessed versus opponent teams) occurrence of technical performance parameters over the 7 assessed matches

\begin{tabular}{|c|c|c|c|c|c|c|c|}
\hline \multirow[b]{2}{*}{ Variable } & \multicolumn{7}{|c|}{ Assessed team score vs opponent team score } \\
\hline & $\begin{array}{c}1^{\text {st }} \\
\text { match }\end{array}$ & $\begin{array}{c}2^{\text {nd }} \\
\text { match }\end{array}$ & $3^{\text {rd }}$ match & $4^{\text {th }}$ match & $5^{\text {th }}$ match & $6^{\text {th }}$ match & $7^{\text {th }}$ match \\
\hline Goal attempts & 6 vs 7 & 7 vs 7 & 4 vs 4 & 10 vs 3 & 10 vs 12 & 4 vs 4 & 3 vs 13 \\
\hline Total passes & 166 vs 142 & $\begin{array}{c}149 \text { vs } \\
168\end{array}$ & 174 vs 183 & 119 vs 213 & 158 vs 137 & 123 vs 186 & 135 vs 139 \\
\hline Successful passes & 128 vs 107 & $\begin{array}{c}112 \text { vs } \\
118\end{array}$ & 128 vs 112 & 77 vs 158 & 118 vs 87 & 76 vs 137 & 95 vs 93 \\
\hline $\begin{array}{c}\text { Unsuccessful } \\
\text { passes }\end{array}$ & 38 vs 35 & 37 vs 50 & 46 vs 71 & 42 vs 55 & 40 vs 50 & 47 vs 49 & 40 vs 46 \\
\hline Total headers & 8 vs 16 & 14 vs 30 & 29 vs 18 & 13 vs 17 & 16 vs 15 & 5 vs 20 & 25 vs 29 \\
\hline $\begin{array}{l}\text { Total tackles } \\
\text { /interceptions }\end{array}$ & 60 vs 62 & 79 vs 147 & 108 vs 163 & 141 vs 130 & 109 vs 102 & 100 vs 95 & 71 vs 75 \\
\hline $\begin{array}{c}\text { Total } \\
\text { involvement }\end{array}$ & 350 vs 334 & $\begin{array}{c}360 \text { vs } \\
454\end{array}$ & 423 vs 424 & 351 vs 469 & 409 vs 343 & 312 vs 375 & 344 vs 322 \\
\hline
\end{tabular}


Figure 1. Resting salivary measures (fasted state) across the investigation period (Mean $\pm \mathrm{SD})$.

Figure 2. Tackles and interceptions over the 7 assessed matches (Mean \pm SD) 